\title{
Interferometric connection of large ground-based telescopes
}

\author{
J.-M. Mariotti ${ }^{1}$, V. Coudé du Foresto ${ }^{2}$, G. Perrin ${ }^{1}$, Peiqian Zhao ${ }^{1,3}$ and P. Léna ${ }^{1,4}$ \\ 1 Observatoire de Paris, DESPA, F-92195 Meudon Principal, France \\ 2 Max-Planck-Institut für Astronomie, Königstuhl 17, D-69117 Heidelberg, Germany \\ 3 Nanjing Astronomical Instruments Research Center, Chinese Academy of Sciences, Nanjing, 210042, China \\ 4 Université Paris 7 - Denis Diderot, Paris, France
}

Received April 11; accepted September 27, 1995

\begin{abstract}
In this article, we argue that the recent advent of two technological breakthroughs in the field of Optics, namely the development of Adaptive Optics and of infrared single-mode fibers, is likely to modify our conception of astronomical interferometry at optical wavelengths. In particular, it should become possible to realize the coherent combination of the giant telescopes that tend to cluster at the best ground-based astronomical sites. Although more limited in their scope than the dedicated interferometric systems, these potential arrays could achieve impressive performances in terms of sensitivity and angular resolution. As an example, we discuss in more details the case of a Mauna Kea cluster of large telescopes.
\end{abstract}

Key words: telescopes — techniques: interferometry — techniques: adaptive optics — instrumentation: interferometers — optical fibers: single-mode — infrared: general

\section{Introduction}

\subsection{Background}

The fact that large facilities for astronomical observations tend to concentrate in a few observatories is not new. One can easily figure out two main reasons which account for this tendency:

1. The cost and the level of technological sophistication of present-day telescopes are such that it is mandatory to erect them in the best available sites. Traditionally, astronomers have always paid attention to the number of clear nights per year. Nowadays, with the introduction of new domains and observational techniques (infrared observations, diffraction-limited imaging, multislit spectroscopy, low sky background photometry,...) many parameters enter in the definition of the 'figure of merit' of a site, e.g., the mean water vapor content, the mean value of the seeing and its statistics, the distributions of wind velocities and directions,.... Consequently, the number of ground-based sites suitable for a modern telescope is small and it can be stated that all these sites are virtually known or will eventually be prospected, including Antartica (Bally 1993).

2. Since these sites tend to be located in high-altitude and desertic regions, the development and maintenance costs for opening a new site tend to be pro-

Send offprint requests to: J.-M. Mariotti (mariotti@obspm.fr) hibitive. Hence, sharing this load between several facilities erected on the same site appears to be a costeffective approach.

As a consequence, several very large observatories, gathering from a few up to a dozen of telescopes, have developed during the recent years, e.g., the observatories at Kitt Peak, Calar Alto, Roque de los Muchachos, Cerro Tololo, La Silla, Mauna Kea...

The idea of taking advantage of the proximity of several large telescopes in such observatories to perform interferometric observations by coherently coupling their beams is not new either: for instance the interferometric coupling of the Canada-France-Hawaii Telescope (CFHT) with the United Kingdom InfraRed Telescope (UKIRT) on Mauna Kea was proposed by Grundmann et al. (1981). Likewise, Léna et al. (1988) suggested to couple interferometrically the La Silla $3.6 \mathrm{~m}$ telescope with its Cassegrain Auxiliary Telescope (CAT). None of these suggestions reached the detailed design phase. One can put forward two arguments to explain the lack of motivation to pursue such proposals

1. It is extremely difficult, and hence extremely costly, to extract the beam from a telescope which may not have been built for this, to transfer it optically over long distances, to adjust its optical path delay and then to recombine it with other beams. The cost is not only financial, but also implies heavy use of prime 
observing time on each individual telescope for system debugging and commissioning.

2. In seeing limited conditions the gain in sensitivity is not decisive between small $(\simeq 0.5 \mathrm{~m})$ and large $(\simeq 4 \mathrm{~m})$ telescopes: it can be shown (e.g., in Roddier \& Léna 1984a, b; Dyck \& Kibblewhite 1986) that the limiting magnitude of an optical interferometer is a steep function of the Fried parameter $r_{0}$ characterizing the strength of the turbulence, but is only weakly dependent on the diameter $D$ of the individual pupils in the array. The actual gain in sensitivity with $D$ is a complex function of the experimental parameters, but it can be as low as $D^{1 / 6}$.

Therefore it was considered much more efficient to build a dedicated interferometer, even with small telescopes, rather than to try to couple large telescopes which had never been designed to provide an interferometric mode.

We claim that these arguments are no more valid.

\subsection{The revolutions of adaptive optics and fiber-linked interferometry}

It is of course well known that Adaptive Optics (AO) considerably increases the sensitivity of an interferometric array by restoring the transverse coherence of the wavefront over its individual pupils (Roddier \& Léna 1984a, b; Greenaway 1992; Mariotti 1993). The efficiency of an AO system is usually expressed in terms of the Strehl ratio $\mathcal{S}$ of the image it produces which is, for a point source, the ratio of the central intensity of the corrected image to the central intensity of an ideal, diffraction limited image of a source with the same magnitude. It can be shown (Conan 1994) that for a single mode interferometer the sensitivity of the array is approximately proportional to the central intensity of the images formed by the telescopes, i.e., to $\mathcal{S} D^{2}$. If the performances of the $\mathrm{AO}$ system is such that the Strehl ratio reaches high values, say $\mathcal{S} \geqq 0.2$, the interferometric combination of large telescopes is considerably favored. This is one of the key arguments for projects like the VLT Interferometer (VLTI) or the Keck Imaging Interferometric Array (KIIA).

Unlike Adaptive Optics, which is likely to revolutionize the whole field of infrared (IR) instrumentation (Ridgway 1993), single-mode optical fiber technology is only relevant to interferometry. The idea of coherently coupling telescopes with optical fibers is more than ten years old (e.g., Froehly 1981), but the experimental demonstration has been obtained only recently (Coudé du Foresto \& Ridgway 1991) in the near infrared. The use of guided optics is an appealing concept for the design of an interferometer: fibers can maintain a constant optical path between two points in motion or in vibration; once the flux is launched inside the fiber it is protected against local seeing and geometrical aberrations; and, last but not least, fibers can extract an optical signal from any point in an optical set-up and transport it easily to any other location.
Thus, fibers can link telescopes with focal plane environments not optically designed for interferometry. There are however some drawbacks to the use of fibers which should be seriously taken into account. We discuss them in the next section.

\section{Technological rationale}

In this section, we present a possible technical strategy for coherent combination of large ground-based telescopes.

\subsection{Injection in the single-mode fiber}

We will not discuss here the technical aspects relevant to Adaptive Optics: several meetings have recently covered this field and we refer the readers to these proceedings (e.g., Alloin \& Mariotti 1993). We will only concentrate on the coupling of a focal plane image into a singlemode fiber. This problem has been studied by Shaklan \& Roddier (1988) who have shown that the coupling efficiency of a perfect Airy disk into the fiber could be as high as $\eta_{\mathrm{c}}=0.78$. This factor is degraded if the telescope pupil has a large central obstruction $d$ (Coudé du Foresto 1994), but the additional loss is less than $10 \%$ if $d / D \leqq 0.2$.

The case of coupling a turbulent image into a fiber has also been studied by Coudé du Foresto (1994): he shows that in condition of partial correction by Adaptive Optics, the coupling efficiency is further degraded by a factor closely linked to the Strehl ratio $\mathcal{S}$ of the image. Recent experimental results (Coudé du Foresto \& Hubin 1995) obtained with an IR single-mode fiber at the corrected focus provided by the COME-ON+ instrument (Rousset et al. 1994) of the ESO 3.6 m telescope of La Silla, have fully confirmed these expectations.

Hence, the sensitivity of an AO-corrected fiber-linked interferometer should be, everything else considered equal, proportional to $\eta_{\mathrm{c}} \mathcal{S} D^{2}$, to be compared with $\mathcal{S} D^{2}$ (cf. Sect. 1.2) for a classical approach. This moderate disadvantage could even be compensated by the elimination of the many reflections necessary to direct the free-airpropagating beam from the coudé foci of the individual telescopes to the delay lines.

\subsection{Beam transportation in single-mode fibers}

By definition, single-mode fibers preserve the phase of the radiations they guide, thus allowing their use in an interferometer. Care should be taken however that the total optical path inside a fiber can slowly evolve under thermal variations and mechanical stress; in the case of hectometric to kilometric fiber lengths that we will consider here, it is thus mandatory to measure, and possibly actively control (Reynaud 1994) the optical path differences between the various fibers of the array. We will come back to this problem in Sect. 2.5. 
Fibers may also alter in two ways the status of the radiation that they guide. Firstly, the transmission of the material used in a given fiber restricts its use to a given wavelength domain. Inside this domain, the fiber will have an attenuation coefficient that will eventually limit the total length of fibers in each arm of an astronomical interferometer. In the visible and near-IR domains (up to $1.6 \mu \mathrm{m}$ ), silica components with virtually no attenuation over kilometric distances have been developed for telecommunication applications: these fibers fulfill the astronomical requirements. Above $2 \mu \mathrm{m}$, the attenuation of silica fibers becomes high, and one should preferably use fluoride glass fibers up to $4 \mu \mathrm{m}$. This material allows to propagate astronomical beams up to about a hundred meters, and gaining a factor of 10 should be technologically achievable during the next few years (Mazé 1994). Above $4 \mu \mathrm{m}$, chalcogenide glass fibers have been developed; to our knowledge, no single-mode component has been commercialized yet, and the attenuation per meter remains very high. For the $10 \mu \mathrm{m}$ domain single-mode hollow wave guides and guided optics components have also been developed (Jenkins et al. 1992), which also show attenuation levels which are presently too high for applications in astronomical interferometry. It is hazardous to predict any impending breakthrough in this domain.

Moreover, fibers are dispersive and the need to use large optical bandwidth for astronomical applications results in tight constraints for the equalization of the lengths of the fibers and of their mean dispersion. These constraints can be relaxed if dispersion-flattened fibers are used. This kind of fibers, for which, around a given wavelength, the wave-guide dispersion compensates the material intrinsic dispersion, have been actively developed in the near-IR domain (at 1.2 and $1.6 \mu \mathrm{m}$ ) for telecommunication applications (e.g., Okamoto et al. 1979), and could probably be made with fluoride glass.

Secondly, a so-called 'single-mode' fiber actually guides two radiation modes, corresponding to two orthogonal polarization states. If care is not taken, mode coupling during the propagation inside the fibers of an interferometer can alter the fringe visibility at the output, or even cancel it. To avoid this problem it is of course possible to select a given polarization state with polarizers at the output of the fibers. Since astronomical sources are usually not polarized, half of the light is lost in this operation. Fortunately, in the recent years, polarization preserving fibers, which avoid this mode coupling problem and allow to use all the transmitted light at the fiber output, have become available, both in the silica and the fluoride glass domains. Usually, the core of these fibers is asymmetric, thus the coupling efficiency $\eta_{\mathrm{c}}$ is lower than for a classical fiber.

\subsection{Delay lines for fiber-linked interferometers}

Fibers can transport the beams from the telescopes to the recombination point of the array, but they cannot compen- sate for the time-varying optical path difference (opd) between the beams that occurs because the entrance pupils of the telescopes do not sit on the same wavefront. To cancel the opds between the various beams, we still need to introduce a variable delay, usually obtained by forcing the light to travel an extra path in a low-dispersion medium (vacuum or air). A useful rule of thumb is that the stroke of such a delay line is about half the length of the baseline (see, e.g., Sect. 4). For a kilometric array, the requirement of building, for each of its baselines, a delay line with several hundreds of meters of stroke and yet a sub-wavelength precision of motion appears prohibitive.

At a given instant however, the opd $\Delta$ between two beams can be very large, but its rate of change is not high: it is given by

$$
\begin{aligned}
\frac{\mathrm{d} \Delta}{\mathrm{d} t} & =\frac{\mathrm{d} \Delta}{\mathrm{d} H} \frac{\mathrm{d} H}{\mathrm{~d} t} \\
& =\left[-L_{X} \sin H \cos \delta-L_{Y} \cos H \cos \delta\right] \frac{\mathrm{d} H}{\mathrm{~d} t}
\end{aligned}
$$

where $L_{X}, L_{Y}, L_{Z}$ are the projection of the baseline vector on the usual reference system (Thompson 1985), $H$ and $\delta$ are the hour angle and declination of the source and $\mathrm{d} H / \mathrm{d} t \simeq 7.310^{-5} \mathrm{rd} . \mathrm{s}^{-1}$. Assuming a baseline of one kilometer, the worst case is for an East-West orientation $\left(L_{X}=0\right)$ and $H=\delta=0$. It yields

$$
\frac{\mathrm{d} \Delta}{\mathrm{d} t}=7.310^{-2} \mathrm{~m} . \mathrm{s}^{-1}
$$

In other words, around a given instant, the delay $\Delta$ can be decomposed into a large DC component (a few hundred meters) and a small $\mathrm{AC}$ variation (a few centimeters per seconds). As already discussed for other long-baseline interferometric projects (Faucherre 1991), we propose to take care separately of these delays.

\subsubsection{The short stroke delay line}

Let us consider a delay line with a maximum delay of a hundredth of the baseline, i.e., in our example, 10 meters. This is about the delay used in high resolution Fourier Transform Spectrometers (Brault 1985) and is much less than, for instance, the stroke of the delay lines of the VLT Interferometer (Faucherre 1991). Although apparently complex, such components are well within the capabilities of present day technology.

Moreover, it has recently been proposed that such delay lines could be realized by mechanically stretching a dispersion-flattened fiber (Coudé du Foresto et al. 1995): in particular, these authors have proposed a solution where a single fiber could tolerate a differential delay of $10 \mathrm{~m}$ while maintaining dispersion inside the whole $\mathrm{K}$ photometric window at a tolerable level.

Fluoride fibers can bear an elongation of at least $1 \%$ of their total length (Mazé 1994) while remaining in the elastic domain. Still, this means that another kilometer of 


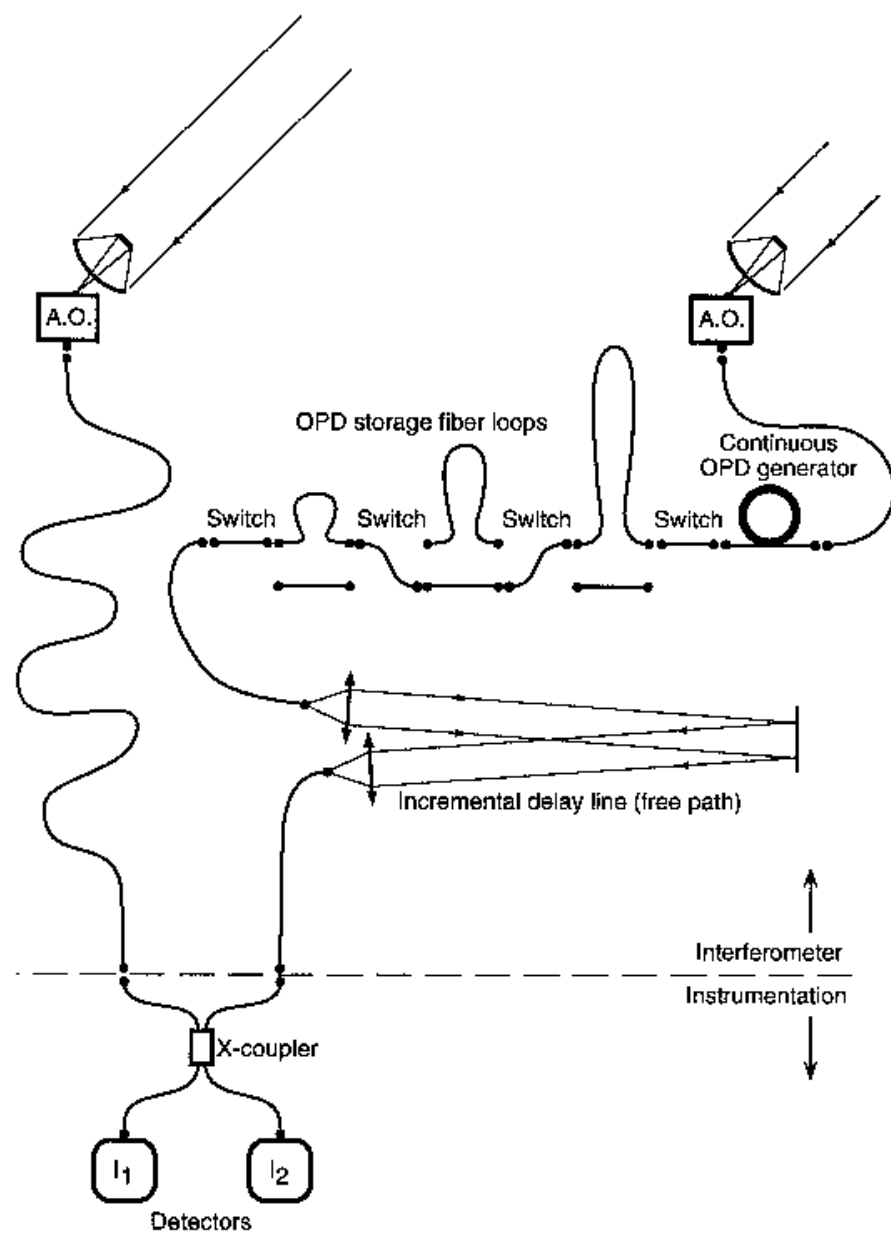

Fig. 1. Interferometer overview: stellar radiation is picked-up by fibers at the adaptive optics corrected foci of two telescopes; radiation goes through a two-stage (continuous and incremental) optical path difference control; the suitably delayed beams are combined in a fibered directional coupler and focused on detectors

fiber would have to be used in order to obtain the required $10 \mathrm{~m}$ of variable delay. To avoid this problem, as well as to reduce the perturbing effects induced on the fiber by the stretching, Zhao et al. (1995) have proposed to use a series of dispersion-flattened fiber loops, whose length would increase as powers of 2 of some unit length, and which could be commuted in or out the fiber path by rapid optical switches: this would reduce the required stroke of the stretched fiber to about this unit length, e.g. $1 \mathrm{~cm}$, and would allow to build a full $10 \mathrm{~m}$ range delay line with ten fiber loops with lengths ranging from 1 to $512 \mathrm{~cm}$.

\subsubsection{The incremental delay line}

The long-stroke incremental delay line could be easily realized with a classical approach, i.e. by translating a miror inside a tunnel. The total length of the tunnel has to be large (one half of the total base length, or one quarter if a double-pass set-up is used) but the mechanical precision can be rather coarse: indeed, during the observations this delay line does not move, since the delay is controlled by the short stroke delay line. When the full stroke of the latter is reached, it has to be reset quickly to its zero point while the incremental delay line is moved to a new position corresponding to the zero point of the continuous delay line.

We stress the point that this mechanical movement does not have to be precise: a correct optical alignment should be preserved, but this can easily be achieved by using servo-mechanisms for pointing the beam coming from the fiber to the center of the retro-reflector which could be itself a simple flat mirror supported on a tip-tilt mount insuring the alignment of the reflected beam toward the exit of the delay line. We believe that the requirement on the mechanical precision should be only of the order of a millimeter. It is of course mandatory that the actual delay obtained with the new position of the mirror be measured with interferometric precision: this can be easily performed by a classical laser-ranging system using a fiducial mark attached to the carriage. Also, we note that since the diameter of the collimated beam traveling in the delay line can be very small the retro-reflector could be an optical piece of reasonable size. Indeed, its diameter should be essentially determined by Fresnel diffraction effects: in this case, the optimal size of the beam, i.e. the size which minimizes the size of the collecting optics after propagation over a distance $L$, is $d_{\text {opt }}=\sqrt{\lambda L}$. For a wavelength of $10 \mu \mathrm{m}$ and for $L=200 \mathrm{~m}$, this yields $d_{\mathrm{opt}}=5 \mathrm{~cm}$. This, as well as the very long total delay, favors the option of a vacuum system rather than an open air delay line.

Of course, during the positioning phase of the incremental delay line, observations have to be suspended. These interruptions could be kept within reasonable limits, say 10 to $20 \mathrm{~s}$, while the contiguous windows of signal acquisition, even with the maximum rate of change of the opd discussed above are, at least, $137 \mathrm{~s}$ assuming a $10 \mathrm{~m}$ range of the continuous delay line and a kilometric baseline.

\subsection{Beam combination and instrumentation}

At the output of the delay lines, the beams can be either directly combined, or fed back into fibers. In the latter case, guided-optics components, such as polarizationpreserving or polarization-separating X-couplers, optical switches,... could be used to perform the beam combination and the bulk of the instrumental set-up. In the former case, classical solutions could be used. We note however that we are here in the case of a single-mode interferometer; hence the notions of pupil and image planes are not relevant. Examples of single-mode beam combination and instrumentation are discussed in more details in the ESO VLT Report No. 65 (1992). 


\subsection{Opd control}

Figure 1 illustrates a conceptual design of the proposed set-up. Since this design involves several components, each performing a specific task, it is extremely important to check for each beam the actual optical path accumulated from the injection point down to the recombination point. This active metrological control probably cannot be performed by a single system, but has to be divided into checks of individual components

1. At the entrance of the delay lines a laser beam could be split into as many beams as telescopes in the array, injected backwards into the fibers, then retro-reflected at the focal plane of the telescopes and collected back at its launching point. The laser wavelength has to be chosen outside the wavelength domain of interest for the astronomical observations, yet within the singlemode range of the fiber, so that it could be retroreflected by, e.g., an anti-transmission coating of the fiber head. Also, this would allow loss-less injection of the control beam into the fibers through highly chromatic X-couplers.

2. The piece of the continuous delay line where the fiber is actually stretched could be easily monitored with a laser and a fringe counting system. The fiber loops which store the opd could be checked in a Michelson set-up against a fixed reference arm. Since those loops would be in a stable and possibly thermally controlled environment, they should not contribute to large opd variations.

3. As already mentioned in Sect. 2.3.2, a laser ranging system could be used for the control of the incremental delay line. In order to avoid differential errors due to offset propagation, the beam of the ranging equipment could be injected in the fiber just before the entrance of the delay line.

4. Finally, the control of the last section, from the output of the delay line to the mixing point in the beamcombiner would of course depend on the technology used, either free propagation, or guided optics. Whatever the choice, solutions comparable to those described above could probably be used. Again, this part of the set-up would lie in a protected environment, so that the control of the opd would not be more complex than for any other interferometer.

The error signals should then be added, the results compared by pairs of beams (i.e. for each baseline) and nulling commands sent to the short stroke delay lines. Perturbations of the passive components (i.e. all except the stretched fiber) are expected to have very low band-passes, so that achieving sub-wavelength accuracy in internal opd nulling should not be too demanding. This scheme for opdcontrol leaves out of the loop the optical path from the telescope pupil to the injection point into the fiber: it is of course feasible to monitor this path, but this would require equipping each telescope of the array with a dedicated high precision control system. We believe that, unless proven absolutely necessary, this procedure is uselessly difficult: indeed, inspections of large classical telescopes have shown that most of them are unexpectedly stable, as far as opd is concerned (Bourlon \& Léna 1990). More precisely, it is likely that the total opd perturbation inside the telescope is at least one order of magnitude smaller than those produced by atmospheric turbulence.

The importance of these atmospherically induced opd fluctuations (the so-called differential piston mode of the turbulence) depends on the seeing (Roddier 1981) and the baseline or the outer scale of turbulence (Ziad et al. 1995); typical $\mathrm{rms}$ values are between 20 and $150 \mu \mathrm{m}$. In Sect. 3.2 we propose a scheme allowing to correct for this last source of opd perturbations.

\section{Scientific rationale}

\subsection{Wavelength coverage}

Presently, our proposal could be implemented only for wavelength domains where dispersion compensated guided optics components have been developed. This leaves only a few very narrow spectral windows inside the near infrared 'silica domain'. But there is no reason why this technology, developed for telecommunication purposes, could not be extended throughout the whole domain of transmission of silica fibers and even to the 'fluoride glass' domain (Coudé du Foresto et al. 1995), thus opening a window from, say, $1 \mu \mathrm{m}$ to $4 \mu \mathrm{m}$.

For shorter wavelengths, the performances of the interferometer would be seriously reduced due to the poorer Strehl intensities achieved by AO correction and by the very scarce sky coverage. Sodium laser beacons (the socalled artificial guide stars) could help, but this would involve equipping all the telescopes of the array with extremely complex and expensive technology, a solution not in the direction of this proposal. Besides, achromatization of the interferometer in the visible could probably be achieved only for very narrow spectral windows.

On the other hand, the thermal infrared region, say from 4 to $20 \mu \mathrm{m}$, is extremely attractive: adaptive optics will achieve nearly perfect correction for a close to $100 \%$ sky coverage. In order to open this wide window, two problems need to be solved.

1. The technology of single-mode guided optics has to be fully developed. In particular, it is difficult to predict if fibers with very low absorption over kilometric distances could be easily manufactured and, if so, at which cost.

2. Assuming that such components will eventually become available, the problem of the instrumental background thermal emission has to be looked at in details. Two sources of background could cause high instrumental emissivity: 
(a) the cladding of the fibers, at ambient temperature, generates background photons which can penetrate and propagate inside the core of the fiber. Fortunately, these cladding modes are weakly coupled and can be easily eliminated: in practice, it is sufficient to tightly wrap and cool the last section of the fiber (say, 1 meter) in front of the detector.

(b) unfortunately, not all the light path is done within guided optics: the incremental delay line is also a potential source of instrumental background since the output fiber will accept all the light emitted by the delay line tunnel within its acceptance cone. In order to reduce this effect, one could imagine cooling, e.g. to liquid nitrogen temperature, the walls of the tunnel, but this will certainly increase the complexity of the system. It is probably more reasonnable to admit that the instrumental emissivity of the interferometer will be high.

\subsection{Field of view}

By definition, a single-mode fiber accepts only a coherent beam étendue of $\sim \lambda^{2}$. In other words, the field of view picked-up by the entrance fibers of the interferometer will be of the order of the size of an Airy disk, whatever the correction achieved by adaptive optics. To this respect, fiberlinked interferometers share the basic properties of radiointerferometers. This could appear as a severe limitation, but one should remember that these interferometers will be highly diluted, i.e., the ratio of the typical baselength to the telescope pupil diameters will be very high. Since the telescopes of the array will not be movable, one must rely on the super-synthesis effect to improve the spatial frequency coverage. In these conditions, it is well known that the imaging capabilities of such arrays are poor, the typical number of independent pixels in the restored maps being of the order of the number of independent baseline visibilities observed. These arrays are hence well suited for very high resolution imaging of compact sources and not for imaging of extended objects already resolved by monolithic telescopes.

However, it is possible to use the same array to observe two or several nearby Airy disk fields: one obvious solution would be to use a set of independent fibers in the focal plane of each telescope, each positioned so as to pick-up an Airy disk field. The corresponding fibers would then feed independent interferometric set-up, allowing the simultaneous observations of several sources in the field in the spirit of the multi-object spectroscopy instrumentation.

The obvious drawback of such a scheme is that it imposes to replicate most of the elements of the interferometer, including the linking fibers, the short stroke delay lines and the opd control systems, thus boosting the complexity and the cost of the interferometer. An elegant alternative would be to feed two (or even more) beams into the same fiber (Fig. 2). Of course, this requires to mul- tiplex the signals in order to be able to disentangle the beams at the output of the set-up. One can imagine easily several solutions:

- Polarization multiplexing, where the beams are polarized at right angles and fed to a polarization preserving fiber. At the output of the delay lines, the beams are easily separated by a polarization splitting X-coupler. This is probably the simplest solution, but also the less flexible.

- Spectral multiplexing, where the beams are optically filtered in adjacent spectral bands. A dichroic Xcoupler or a spectrograph can be used to demultiplex the beams at the output. This solution is more flexible, since the optical bandwidth can be somehow adjusted to optimize the performances of the set-up.

- Frequency multiplexing, where one of the interferometric signal is modulated at high frequency by, e.g., modulating the opd with an amplitude $\lambda$ or a few wavelengths. This solution is the most efficient in terms of sensitivity since it allows to conserve all the light, but requires to feed the beams through the same instrumentation, since the demodulation can be performed only after the detection of the interferometric signals.

- Temporal multiplexing, where the beams are increasingly delayed with a group delay of $n \Delta t / 2$, where $n$ is the order of the interferogram and $\Delta t$ is the temporal window of acquisition. This scheme preserves the total polarization, the spectral range and the metrology.

Whatever the solution, since the sources lie at slightly different coordinates, a differential delay appears between the zero opds. In order to remove it, a short stroke delay line can be inserted in one of the path before the mixing point, in a scheme similar to the one proposed by Beckers (1991). This differential delay is $B . \alpha$, where $B$ is the apparent baselength and $\alpha$ is the angular separation projected on the direction of the baseline. For $B=$ $1 \mathrm{~km}$ and $\alpha=1 \mathrm{~min}$ of arc, this leads to $\sim 0.3 \mathrm{~m}$, a delay which is within the stroke of the guided optics delay lines discussed above.

Beside the gain in observing time, we see two obvious applications of this mode of operation:

1. One can imagine for instance implementing a coherencing and/or a cophasing mode, i.e., using a bright reference source to obtain in real time the atmospheric and instrumental phase errors and provide the corrections for the faint sources lying inside the isoplanatic patch surrounding the reference. Since most of the path inside the interferometer will be common to all the sources, the differential errors in opd will be contributed essentially by the angular decorrelation of the atmospheric differential piston. An alternative to this real-time solution would be to use the same set-up, but in a much cheaper open loop version to simply monitor the atmospheric opd variations. The monitored opd error signal is recorded with the pistoned interferogram 


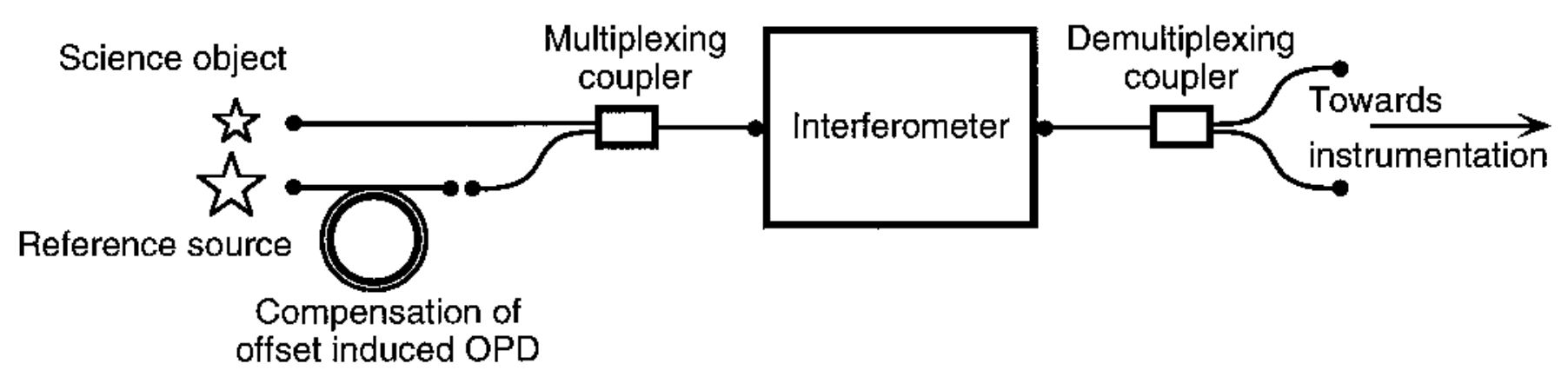

Fig. 2. Multiplexing scheme for feeding several beams into the same fiber

and can be used during the data reduction process to correct the visibility measurements.

2 . In the cophasing mode, the measurement of the differential delay between the zero opds of two sources provides a measurement of the angle between the sources with interferometric precision. This is known as differential interferometric astrometry. The precision in separation determination in this mode has been derived by Shao \& Colavita (1992). Extrapolating their results to a kilometric baseline, we find an accuracy of $\sim 3.5$ micro-arcsec for a source-reference separation of $15^{\prime \prime}$ and an integration time of 1 hour. For comparison, the reflex motion of the Sun due to Jupiter, as seen from $10 \mathrm{pc}$, produces an apparent displacement of \pm 500 micro-arcsec, while the influence of the Earth would amount to \pm 0.3 micro-arcsec.

\subsection{Sensitivity and sky coverage}

The limiting magnitudes and signal to noise ratios (SNR) for interferometric arrays with large adaptive opticscorrected telescopes have been derived by several authors. Since those results show a general agreement, we can refer to the case of the VLTI (ESO 1989; Beckers 1991). With respect to these estimates based on a classical optical approach, we believe that a fiber-linked interferometer would not be much less sensitive: the main factors that will determine the overall transmission efficiency $\tau$ are the coupling efficiency of the free air propagating beam into the focal plane fiber $(\sim 0.6)$, the transmission of the kilometric fiber $(\geq 0.95)$, the fiber-to-fiber coupling efficiency through the incremental delay line (unknown but $\geq 0.6$ ), the coupling efficiencies at fiber connections $(\geq 0.98$ per connector and optical switches) and the transmission of the X-couplers $(\geq 0.97)$. Although highly uncertain, we believe that a safe estimate of $\tau$ is between 0.1 and 0.2 . This is to be compared with the 0.5 estimate for the 20-mirror optical train of the VLTI (ESO 1989), but we stress that in the latter case influence of dust scattering on the mirrors, ageing of the coatings and imperfect alignments have not been take into account. Everything else equal, the fiber-linked ver- sion of an optical interferometer as complex as the VLTI or the KIIA would represent a loss of 1 to 1.5 in limiting magnitudes with respect to its 'classical' counterpart. Given the opportunity opened by these giant interferometric arrays, this disadvantage does not appear large.

The price to pay in terms of sky coverage in the off-axis co-phased mode is more severe: assuming that the limiting magnitude for fringe tracking for a classical interferometer with $8 \mathrm{~m}$ telescopes is $\sim 16$ (Mariotti 1993), a safe estimate for a fiber-linked interferometer would be 14.5. The sky coverage for such a limiting magnitude depends on the isoplanatic angle for the differential piston, which in turn depends on the mostly unknown outer scale of turbulence $\mathcal{L}_{0}$ (Ziad et al. 1995) and on the wavelength of observation: it is likely to be quite large in the thermal infrared, but vanishingly small in the visible. Again, sodium laser beacons may improve the situation (Rigaut \& Mariotti 1995) but this is beyond the scope of this paper.

\subsection{A preliminary scientific program}

The scientific importance of high resolution observations provided by large ground-based arrays of telescopes for the astrophysics of the next century has been already discussed in details in recent books and conferences. It provides the rationale for ambitious projects such as the VLT interferometric mode (referred to as VLTI) and the coherent combination of the two 10-m Keck telescopes (referred to as KIIA for Keck Imaging Interferometric Array). Obviously, the same broad range of astrophysical programs, from solar system bodies to high redshift galaxies, could be considered for our proposal. We note however that a fiber-linked array of giant telescopes would have a narrower spectral coverage and slightly reduced sensitivity (Sect. 3.3), but could provide a better imaging quality (if more than four telescopes are included) and a gain in angular resolution of an order of magnitude. With this in mind, we can focus on three particularly promising programs

1. Although it has been a very active field of research, both from a theoritical and an observational point of 
view, the final elucidation of the physical status of Young Stellar Objects (YSOs) is still under debate. In particular, the paradigmatic picture of a $\mathrm{T}$ Tau star, with its dust and gas accretion disk and its highly collimated jets, has been built through strong indirect evidences. However, actual observations of these disks and jets require high angular resolution techniques. At $2.2 \mu \mathrm{m}$ a $1 \mathrm{~km}$ interferometric array would resolve 0.5 milli-arcsec, i.e. $\sim 0.07 \mathrm{AU}$ at the distance of the closest concentrations of YSOs $(150 \mathrm{pc})$ : hence, the very inner part of the accretion disk, close to the stellar surface could be imaged, as well as the base of the jets. At larger distances from the central star, voids and unstabilities in the disk could be detected. Close young stellar binaries could be resolved and followed during their orbit, leading to mass estimations of the components.

A related subject is the disks around Main Sequence stars (such as $\beta$ Pic). Here again, the interferometer could resolve details in the disk, e.g. voids caused by newly formed planets, but the contrast with respect to the central star imposes to observe at the largest possible wavelengths (Lagage et al. 1994). Taking $\beta$ Pic itself as an exemple, it is interesting to note that the stellar disk $(\sim 1$ milli-arcsec) will be fully resolved, while an hypothetical planet transiting over the star (Lecavelier des Etangs et al. 1995) would not.

In both cases, a large number of such sources could be observable.

2. At the other end of the star's life, when it has left the Main Sequence, the mass loss phenomenon starts to play a significant role. Dust forming in this strong stellar wind gives rise to an IR excess and can eventually hide the star itself at visible wavelengths. If it is currently believed that the grain-gas coupling in the circumstellar envelop can explain the dynamics of the mass outflow at large distances, the detailed mechanism initiating the mass loss close to the stellar surface where the temperature is still too high for dust to form is still under debate. Infrared observations at both high angular and high spectral resolution could allow to directly probe this region where outward motions and shock fronts are likely to play a key role (Lafon 1994). This will be possible by combining the high sensitivity of the array with its high angular resolution (at $1 \mathrm{kpc}$, the size of the stellar disk is about 1 milli-arcsec). Again, this class of objects will provide numerous targets.

3. Finally, a very promising program is related to the study of galactic nuclei, whether active or not. It has been already emphasized that an hectometric array such as the VLTI or the KIIA will allow at $2.2 \mu \mathrm{m}$ to detect in a few years the orbital motions of the group of stars closest to the Galactic Center (ESO 1989, Eckart et al. 1993) thus allowing to directly measure the mass of the central core. Gaining an order of magnitude in resolution will of course allow to achieve the same result faster, but could also open access to similar measurement in external galaxies. Turning our attention to these galaxies, high priority targets would be starburst galaxies and active galactic nuclei (AGNs). At a distance of $10 \mathrm{Mpc}$ for instance, a resolution of 0.5 milli-arcsec translates into $0.02 \mathrm{pc}$, or about one lightmonth: although the central engine will remain unresolved at this scale its immediate surrounding (the broad and narrow line regions and the hot dust component) could be studied in details.

Also in the extra-galactic domain, two programs might have important cosmological implication: such an interferometer would allow to directly determine the distance of external supernovae by monitoring the expansion of the associated IR echo (Chalabaev 1994) and studying the distribution of dark matter in the Universe through the gravitational lens phenomenon (Surdej 1994).

\section{The case of the Mauna Kea observatory}

The site of Mauna Kea on the Hawaii island is certainly one of the best ground-based sites for astronomical observations. Among the large observatories, it has the highest elevation $(\sim 4200 \mathrm{~m})$ and consequently both infrared transmission and seeing statistics are exceptional. Its location at latitude $\sim 20^{\circ} \mathrm{N}$ allows the observation of the whole Northern sky and a large fraction of the Southern one.

Table 1. Large telescopes in operation or in construction at the Mauna Kea site (adapted from the Information Bulletin 19 of the Institute for Astronomy, University of Hawaii, Sept. 1994)

\begin{tabular}{lcc}
\hline Telescope & Diameter $(m)$ & Operational \\
\hline UKIRT & 3.8 & 1979 \\
CFHT & 3.6 & 1979 \\
Keck I & 10 & 1992 \\
Keck II & 10 & 1996 \\
JNLT - Subaru & 8 & 1999 \\
Gemini & 8 & 1999 \\
\hline
\end{tabular}

By the end of this century, Mauna Kea will host four 8-m class telescopes, in addition to the two 4-m class telescopes already in operation as well as several smaller ones. Table 1 lists the six largest of this group, amounting for a total of $\sim 280 \mathrm{~m}^{2}$ of collecting area. In terms of gathering power, the only comparable site will be Paranal (Chile) with the four 8-m telescopes of the VLT $\left(\sim 200 \mathrm{~m}^{2}\right)$ which features an interferometric mode (VLTI).

For each of the six telescopes of Table 1 an Adaptive Optics corrected focus is scheduled. As a matter of fact, a 


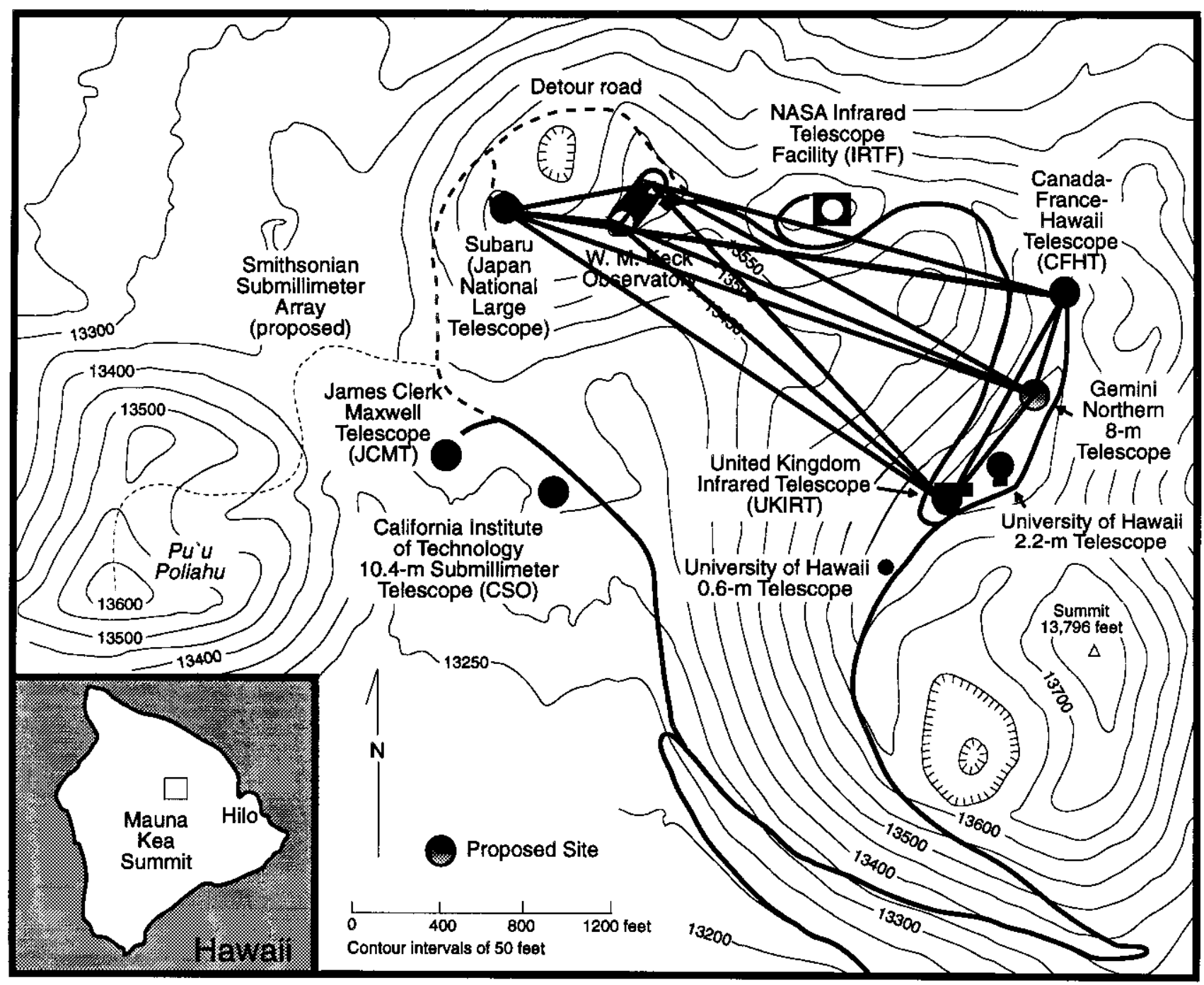

Fig. 3. Map of the summit of the Mauna Kea showing the main telescopes (adapted from the Information Bulletin 19 of the Institute for Astronomy, University of Hawaii, Sept. 1994). The horizontal scale and the elevation are in feet

team of the University of Hawaii has recently developed an $\mathrm{AO}$ system and achieved excellent image correction performances at the CFHT and UKIRT (Roddier et al. 1994).

Figure 3 is a map of the Mauna Kea summit showing the location of the main observing facilities. We have also drawn on the map the 15 baselines corresponding to the combination of the six telescopes of Table 1. The shortest baseline $(75 \mathrm{~m})$ corresponds to the combination of Keck I and Keck II, while the longest reaches $\sim 800 \mathrm{~m}$. Unfortunately, the two 8-m telescopes and the group of the two Kecks lie approximately on an $\mathrm{E}-\mathrm{W}$ line. It is hence mandatory to include in the array the two 4-m telescopes which provide together with Gemini some N-S baselines. This is illustrated in Fig. 4 which gives the spatial frequency coverages obtained for various source declinations. For each map, the tracks have been stopped for zenithal distances greater than $60^{\circ}$. The purpose of these maps is only for illustration: they have been computed from direct estimates in Fig. 3 of the $(X, Y, Z)$ coordinates of the telescopes: hence they are accurate to only $\sim 5 \%$.

Figure 5 shows the corresponding Point Spread Functions. In these computations we have taken into account the actual diameter of the telescopes for each baseline. It is noteworthy that Michelson interferometry is rather tolerant with respect to a photometric unbalance of the combined beams: Roddier \& Léna (1984a, b), then Beckers (1988), have shown that, assuming diffraction-limited beams, the sensitivity of a telescope pair with unequal apertures diameters $D_{1}$ and $D_{2}$ is proportionnal to $D_{1} D_{2}$, i.e. is equivalent to the sensitivity of an identical telescope pair of diameter $\delta=\sqrt{\overline{D_{1} D_{2}}}$. In the case of AO-corrected telescopes, it is easy to show that the interferometric 

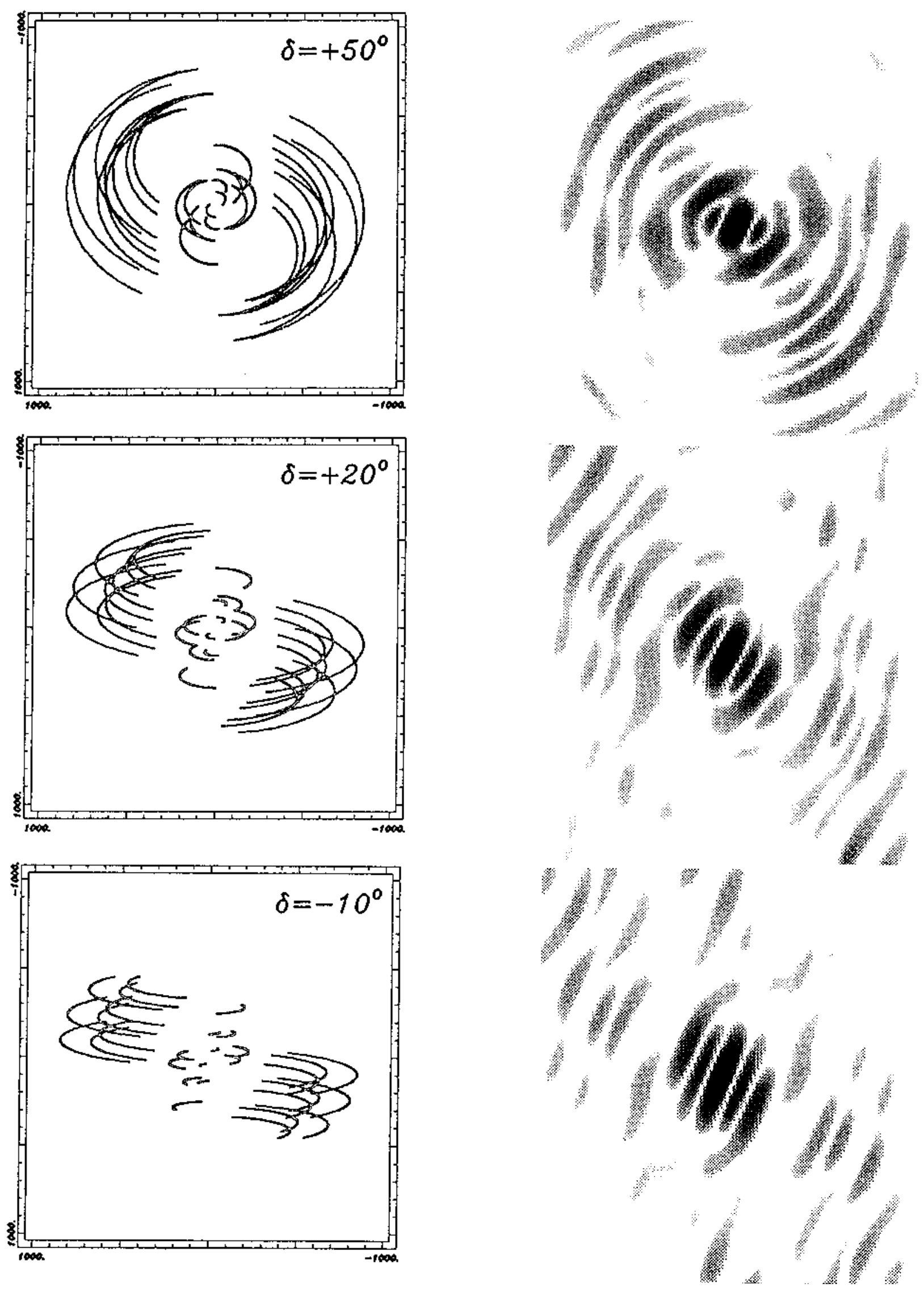

Fig. 4. Spatial frequency coverages for the interferometric combination of the six largest telescopes of Mauna Kea and various source declinations. The scales are in meters

Fig. 5. Point Spread Functions corresponding to the $(u, v)$ coverages of Fig. 4 (in log scale) 

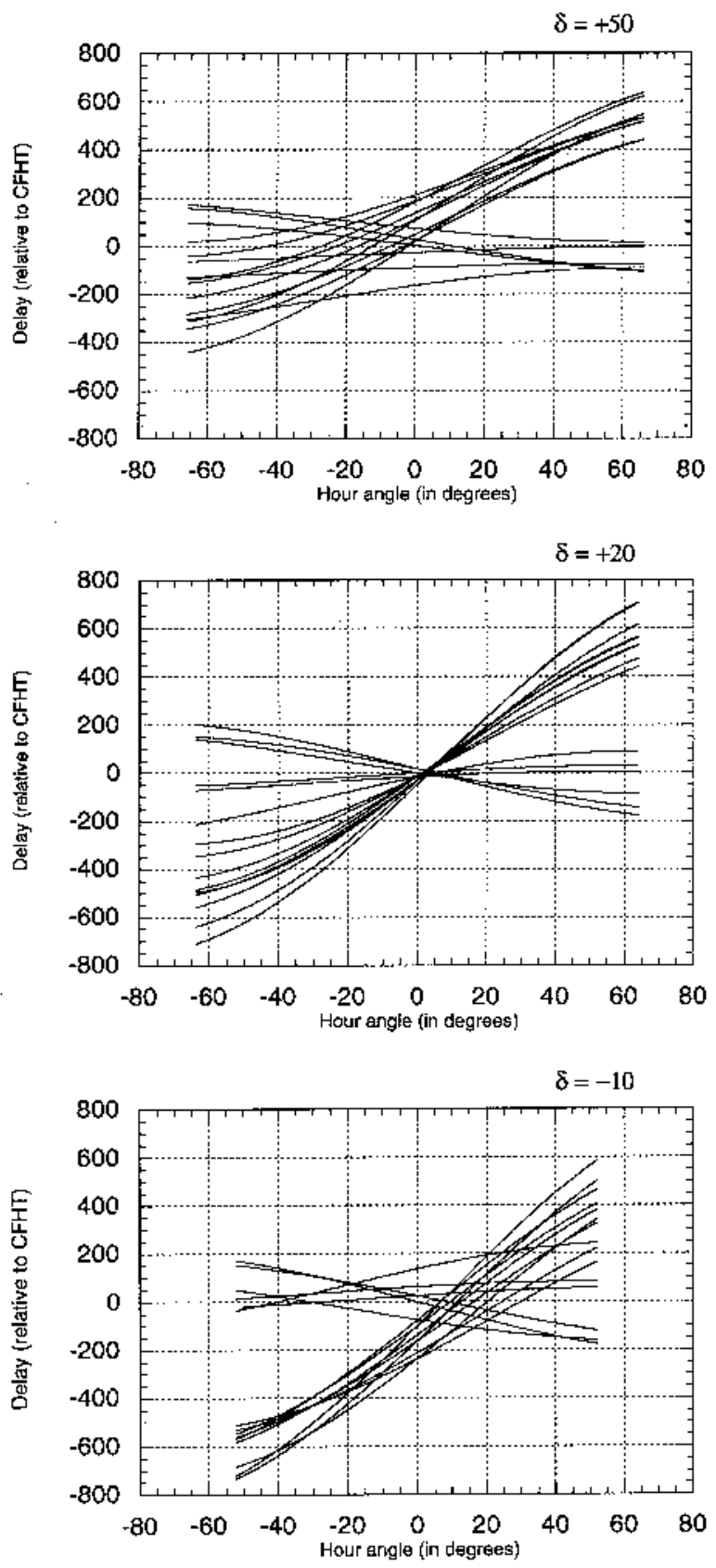

Fig. 6. Relative delays $w$ corresponding to the $(u, v)$ coverages of Fig. 4

signal is proportionnal to $\sqrt{\mathcal{S}_{1} \mathcal{S}_{2}} D_{1} D_{2}$, where the subscripts refer to the telescopes of unequal size. Now, for identical conditions of turbulence, the dependency of $\mathcal{S}$ on $D$ is not obvious: if we assume that the AO systems are dimensioned with respect to the wavefront (i.e., same spatial sampling or, the greater the telescope, the greater the number of degrees of freedom), and optimal correcting conditions (i.e., infinite bandpass, no wavefront analysis noise), then the residual wavefront phase variance after correction $\sigma_{\phi}^{2}$ can be assumed to be the same for all the telescopes. It also means that the 'coherent energy' as defined by Conan (1994) $E_{\mathrm{c}} \simeq \exp -\sigma_{\phi}^{2}$ is independent of the telescope diameter $D$. For reasonnable conditions of correction, say $\mathcal{S} \sim 0.2$, we can use the approximation $E_{\mathrm{c}} \simeq \mathcal{S}$. Hence, in such conditions, the conclusion reached by Beckers (1988) would also hold: for instance, combining one of the Keck telescopes with the CFHT yields an effective interferometric aperture of $2 \times 6 \mathrm{~m}$. In practice, one should take into account the real experimental conditions: for instance, for photon-starved wavefront analysis, the large telescopes are favoured since their estimation of the low orders of aberration benefits from the larger collecting area. On the other hand, it is likely that smaller telescopes will be equipped with AO-systems somewhat over-dimensioned with respect to the larger ones. Only numerical simulations can tell if these effects will compensate each other or not. For the computations leading to Fig. 5 we have assumed $\delta=\sqrt{D_{1} D_{2}}$.

Finally, Fig. 6 illustrates the delays $w$ between all the telescope pairs of the array for the several source declinations. The maximum total delay is about $1200 \mathrm{~m}$. But it is easy to switch, when $w=0$, the two fiber outputs between the continuous and incremental delay lines. This allows to use twice a shorter delay line. Finally, the required stroke for the incremental delay line is $300 \mathrm{~m}$, or $150 \mathrm{~m}$ if a double-pass is used. This is about the length of the VLTI delay lines tunnel (Faucherre 1991), but we again emphasize that the incremental concept described above requires far less room and far less mechanical precision than the classical solution.

\section{Conclusions}

The development of adaptive optics opens the way to high sensitivity interferometric observations by coherent coupling of large telescopes. We have shown that on-going progress in the field of guided optics will allow to link isolated telescopes on the same site, provided that these telescopes are corrected by adaptive optics. Our proposal does not result in heavy investments in order to implement this interferometric mode: the most demanding, and probably quite expensive, constraint would be to build on the site a few hundred meters long tunnel to protect the incremental delay lines and a laboratory for the coherent focus environment.

Yet, a research and development program should be actively started in the field of guided optics in order to improve or to build prototypes of crucial components. We emphasize three of them:

- null-dispersion fibers have been produced for wavelength domains relevant to the telecommunication problem. It remains to extend this concept to other domains, in particular the near-IR. If these components can be manufactured, they would allow to adopt 
a guided-optics solution for the continuous delay lines. It is interesting to note that in this configuration none of the components of the interferometer will move during the observations.

- developing guided-optics solution for the thermal IR (4 to $12 \mu \mathrm{m}$ ) would allow to extend the scientific coverage of the interferometer. Technically, this domain is extremely interesting, since it corresponds to the best performances of adaptive optics correction. In particular, the isoplanatic angle, and consequently the sky coverage, will be large.

- low noise high quantum efficiency detectors will be mandatory to achieve the highest sensitivity of this interferometric mode. But of course, this point is not specific to fiber-linked interferometers.

The scientific potential of these giant interferometers is so exceptional that we believe that this will foster the collaboration of the teams managing the individual telescopes of the potential array. In particular, we emphasize that 'modest size' telescopes, e.g. 2 to 4-m class telescopes, should not be dismissed, since their coupling with larger ones is increasing the total number of baselines of the array with comparable sensitivities. This is in particular true if these baselines provide, as it is the case for the Mauna Kea array, complementary spatial frequency coverages. Also, it will be far less demanding to demonstrate the feasibility of this technique and to show its scientific value by starting to couple the less over-subscribed telescopes of the site.

Finally, it could be suggested that the precise location of a new telescope project for those large observatories should be examined with respect to a possible optimisation of the $(u, v)$ plane coverages.

Acknowledgements. This work has been supported by the CNES and the CNRS through its ULTIMATECH programme. It is also a pleasure to thank G. Mazé (Le Verre Fluoré) for his constant support and Jean-Pierre Véran for technical assistance.

\section{References}

Agabi A., Borgnino J., Martin F., Tokovinin A., Ziad A., 1995, A\&A (in press)

Alloin D., Mariotti J.-M., 1993, Proceedings of the NATO ASI "Adaptive optics for astronomy", Cargèse (Corsica), Series C: Vol. 423. Kluwer Academic Publishers

Bally J., 1993, "The cold universe", XIIIth Moriond astrophysics meetings. In: Montmerle T., Lada C.J., Mirabel I.F., Trân Thanh Vân J. (eds.), Les Arcs (France), 13-20 March 1993

Beckers J.M., 1988, Proceedings of the NATO ASI "Diffraction-limited imaging with very large telescopes". In: Alloin D., Mariotti J.-M. (eds.), Cargèse (Corsica), Ser. C: Vol. 274. Kluwer Academic Publishers

Beckers J.M., 1992, Exp. Astron. 2, 57

Bourlon P., Ducros T., Faucherre M., 1991, "High-resolution imaging by interferometry II". In: Beckers J. \& Merkle F. (eds.), Garching (Germany), p. 1215
Brault J.W., in: "High resolution in astronomy", 15th advanced course of the Swiss Society of Astrophysics and Astronomy, Saas-Fee (Switzerland)

Chalabaev A., in: ESO workshop on "Science with the VLT", Garching (Germany), June 1994

Conan J.-M., 1994, PhD thesis, Université Paris 11

Coudé du Foresto V., 1994, PhD thesis, Université Paris 7 Denis Diderot

Coudé du Foresto V., Hubin N., 1995 (in preparation)

Coudé du Foresto V., Ridgway S., 1991, "High-resolution imaging by interferometry II". In: Beckers J. \& Merkle F. (eds.), Garching (Germany), p. 731

Coudé du Foresto V., Perrin G., Boccas M., 1995, A\&A 293, 278

Dyck H.M., Kibblewhite E.J., 1986, PASP 98, 260

Eckart A., Genzel R., Hofmann R., Sams B.J., Tacconi-Garman L.E., 1993, ApJ 407, L77

ESO VLT Report No. 59: "The VLT Interferometer", 1989

ESO VLT Report No. 65: "Coherent combined instrumentation for the VLT Interferometer", 1992

Faucherre M., 1991, "High-resolution imaging by interferometry II". In: Beckers J.M. \& Merkle F. (eds.), Garching bei München (Germany), p. 1179

Froehly C., 1981, ESO Conf. on "Scientific Importance of High Angular Resolution at Infrared and Optical Wavelengths". In: Ulrich M.H. \& Kjär K. (eds.), Garching (Germany), p. 285

Greenaway A., 1992, ESA workshop on "Targets for SpaceBased Interferometry". In: Volonté S. (ed.), Beaulieu (France), p. 43

Grundmann W.A., Odgers G.J., Richardson E.H., 1981, ESO Conf. on "Scientific Importance of High Angular Resolution at Infrared and Optical Wavelengths". In: Ulrich M.H. \& Kjär K. (eds.), Garching (Germany), p. 253

Jenkins R.M., Devereux R.W.J., Heaton J.M., 1992, Opt. Lett. 17,991

Lafon J.-P.J., 1994, in: ESO workshop on "Science with the VLT", Garching (Germany), June 1994

Lagage P.-O., Pantin E., 1994, Nat 369, 628

Lecavelier des Etangs A., Deleuil M., Vidal-Madjar A., Ferlet R., Nitschelm C., Nicolet B., Lagrange-Henry A.-M., 1995, A\&A (submitted)

Léna P., Ridgway S.T., Mariotti J.-M., 1988, "High-resolution imaging by interferometry". In: Merkle F. (ed.), Garching (Germany), p. 1039

Mariotti J.-M. 1993, see Alloin \& Mariotti 1993

Mazé G., 1994 (private communication)

Okamoto K., Edahiro T., Kawana A., Miya T., 1979, Electron. Lett. 15, 729-731

Reynaud F., Alleman J.J., Connes P., 1992, Appl. Opt. 31, 3736

Ridgway S.T., 1993, see Alloin \& Mariotti 1993

Rigaut F., Mariotti J.-M. (in preparation)

Roddier F., 1981, Progr. Opt. XIX, 281-376

Roddier F., Léna P., 1984a, J. Opt. 15, 171

Roddier F., Léna P., 1984b, J. Opt. 15, 363

Roddier F., Anuskiewicz J., Graves J.E., Northcott M.J., Roddier C., 1994, in: SPIE Conf. 2201 "Astronomical Telescopes and Instrumentation for the 21st century". In: Ealey M.A. \& Merkle F. (eds.), Kona (Hawaii), p. 2 
Rousset G., Beuzit J.-L., Hubin N., et al., 1994, SPIE Conf. 2201 "Astronomical Telescopes and Instrumentation for the 21st century". In: Ealey M.A. \& Merkle F. (eds.), Kona (Hawaii), p. 1088

Shaklan S., Roddier F., 1988, Appl. Opt. 27, 2334

Shao M., Colavita M.M., 1992, A\&A 262, 353

Surdej J., 1994, in: ESO workshop on "Science with the VLT",
Garching (Germany), June 1994

Thompson A.R., 1985, in "Synthesis Imaging", NRAO Summer School, Socorro (New Mexico)

Zhao P., Mariotti J.-M., Coudé du Foresto V., Léna P., Perrin G., 1995, in SPIE Conf. 2476 "Fiber optics in astronomical applications", Orlando (Florida), 17-21 April 1995 